\title{
Tooth impaction: A new variable in difficulty index of third molar extraction
}

\author{
MR Jaisaini, A Dongol, P Acharya, AK Yadav \\ Department of Oral and Maxillofacial Surgery \\ B.P. Koirala Institute of Health Sciences, Dharan, Nepal
}

\begin{abstract}
Third molar surgery is one of the major events carried out world-wide in a maxillofacial clinic. Preoperative assessment of surgical difficulty is fundamental to planning of extraction of impacted third molars. Various models for this assessment have been proposed but none is universally applicable. Although several studies have identified a plethora of factors associated with extraction difficulty, as measured via a wide variety of proxy variables, still, there are conflicting reports and wide variations of the enumerated factors. Adding to the existing knowledge, through this communication we would like to suggest that tooth impaction should also be considered as a variable of difficult third molar extraction. It requires both surgical expertise and added operating time thus affecting difficulty index.

So we suggest tooth impaction should be considered as a difficulty assessment tool and studies be carried out to produce an evidence based database.
\end{abstract}

Key words: Difficulty index, tooth impaction, third molar surgery

\section{Introduction}

Third molar surgery corresponds to a significant proportion of surgical event carried out world wide as a part of contemporary oral and maxillofacial surgery. Thus, these surgical procedures should be planned and executed according to the available scientific evidence. Both the patient and dentist must

\section{Address for correspondence}

Dr. Mehul Rajesh Jaisaini

Associate Professor

Department of Oral and Maxillofacial Surgery

B.P. Koirala Institute of Health Sciences, Dharan

E-mail: jaisani_mehul@live.in

Phone: 00977-9852027325 have scientific evidence based information concerning the estimated level of surgical difficulty of every case. ${ }^{1}$ It is important that a paradigm for factors associated with third molar extraction difficulty be developed to serve patients more effectively, to plan operation, and to educate students and residents. $^{2}$ Historically, there have been various efforts at determining are liable model for this assessment. Although many have been postulated, none could be said to be universally impeccable. ${ }^{3}$ The first attempt to develop a model of this nature was made by 
MacGregor in $1976 .{ }^{4}$ Other such widely advocated models are the Winter's, Pell and Gregory's, Pederson's, and the WHARFE system of MacGregor classification/scoring system. Recent literature may have reduced the dearth of information on the estimation of third molar surgical difficulty; still there are conflicting reports and wide variation of the enumerated factors. ${ }^{3}$

Various radiographic and non-radiographic (surgical, demographic) variables have been studied as variables of difficulty of impacted third molar extraction. Mandibular third molars are more difficult to extract than their maxillary counterparts. It is very likely that this effect is due to the greater cortical bone density in the mandibular arch relative to that in the maxillary arch and the additional caution required avoiding IAN injury. As surgical procedure changes from no incision (erupted) to incision required (erupted or impacted), and as depth of impaction increases from soft tissue to full bony, a greater degree of surgical manipulation is required for extraction, thereby prolonging operating time and, by proxy, difficulty. Deviation of teeth from a vertical alignment increases operating time, with distoangular teeth requiring the most time for extraction; this is likely due to the difficulty of crown/root access in horizontal and distoangular teeth compared with vertical and mesioangular teeth. As tooth morphology becomes more favorable, root access and crown positioning are more conducive to extraction and extraction time decreases. It is an intuitive result that increasing surgical experience decreases extraction time-experienced surgeons will have performed more extractions and will have a higher skill level than those with less experience or those in training. $^{2}$

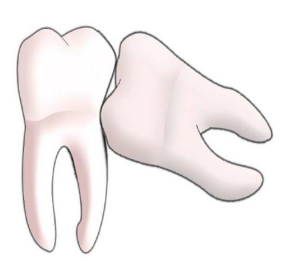

Fig. 1

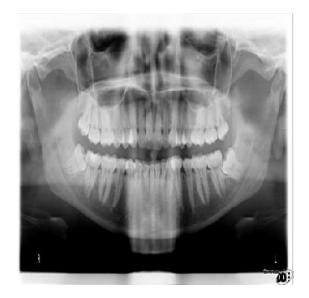

Fig. 2
Fig. 1: Tooth Impaction

Fig. 2: OPG showing bilateral tooth impaction in impacted mandibular third molar.

Adding to the existing list of variable that influence third molar extraction difficulty we suggest that tooth impaction should also be considered as one of the assessment variable. Tooth impaction, a condition in which third molar crown is locked below the greater curvature of second molar crown [Fig 1], also adds to difficulty of third molar extraction. Tooth impactions are often present with mesioangular or horizontally impacted third molars and especially adding to difficulty with extraction in Pell and 
Gregory's level B and level C impactions [Fig 2].

Surgical difficulty is determined by the surgical technique employed and the length of surgery time. ${ }^{2}$ This condition is an added difficulty because it requires a different sectioning technique and expertise than that advocated in conventional sectioning technique. Sectioning technique adds to difficulty because of limited access to the region as well as the third molar crown requires multiple sections in order to relieve the lock, which further adds to operating time. None of the studies published in the literature have taken this condition into account. Given the scarcity of scientific evidence on the proper conduct during the surgical removal of impacted third molars, studies are needed to confirm the main variable of an accurate model for the preoperative prediction of surgical difficulty. ${ }^{5}$ So we suggest tooth impaction should also be considered as a difficulty assessment tool and studies be carried out to produce an evidence based database.

\section{References}

1. Gbotolorun OM, Arotiba GT, Ladeinde A: Assessment of Factors Associated With Surgical Difficulty in Impacted Mandibular Third Molar Extraction. J Oral Maxillofacial Surgery 2007;65: 19771983 ,

2. Susarla SM, AB, and Dodson TB: Risk Factors for Third Molar Extraction Difficulty. J Oral Maxillofacial Surgery 62:1363-1371, 2004

3. Akadiri $O A$, and Obiechina $A E$ : Assessment of Difficulty in Third Molar Surgery- A Systematic Review. J Oral Maxillofacial Surgery 67:771-774, 2009

4. Macgregor AJ: The radiological assessment of ectopic lower third molars. DDSc thesis, Leeds, UK, University of Leeds, 1976

5. Carvalho RWF, and do Egito VasconcelosBC: Assessment of factors associated with surgical difficulty during removal of impacted lower third molars. J Oral Maxillofacial Surgery. 2011; 69:27:14-2721 